\title{
HIGH RESOLUTION SEA ICE DRIFT ESTIMATION USING COMBINED TERRASAR-X AND RADARSAT-2 DATA: FIRST TESTS
}

\author{
Anja Frost, Sven Jacobsen, Suman Singha \\ German Aerospace Center (Deutsches Zentrum für Luft- und Raumfahrt; DLR) \\ Maritime Safety and Security Lab, Bremen, Germany \\ Anja.Frost@dlr.de
}

\begin{abstract}
High resolution sea ice drift fields, the location and extend of converging and diverging zones as well as ice ridges are most important parameters for ship navigation in ice infested waters. In this paper, we present the prototype of a new processor which is aimed to derive the surface ice parameters on the basis of pairs of space-borne Synthetic Aperture Radar (SAR) data of the same and of different sensors, i.e. from data of different bands, resolutions, and orbits. The study is carried out on image data collected during a joint campaign with the Office of Naval Research (ONR) in the western Arctic in 2015. The algorithm proposed is foreseen to be integrated into near-real time (NRT) processing chain at DLR ground stations in order to provide time-critical information as soon as possible to users and stakeholders.
\end{abstract}

Index Terms - Sea ice velocity, SIUV, ship navigation, block matching, phase correlation

\section{INTRODUCTION}

Synthetic Aperture Radar (SAR) sensors are commonly used to observe polar regions - in fact satellite missions such as CryoSat are primarily dedicated to monitor the Arctic and Antarctic. While parameters like sea ice coverage, sea ice type and thickness are one focus, another major topic is the quantification of the sea ice drift. The latter property is most important for ship traffic in ice infested waters, as the data provides a basis of ice drift forecasts. This data is crucial not only to reduce the number of ships trapped in the ice, but also to optimize ships' courses to account for the predicted ice situation and save journey time and fuel. Coarse ice drift prognosis can be calculated on the basis of ambient weather conditions such as wind and sea state. High resolution ice drift maps, however, show spatial differences in the ice drift fields and expose converging and diverging ice areas. They thus can be used to characterize open water leads that are likely to open up or close and to identify regions of compressing ice, which might form ice ridges. Particularly ice ridges are serious obstacles for ships and sometimes hard to overcome even for the largest ice breakers.

A set of high resolution space-borne SAR images provide the opportunity to identify and recognize ice areas and to deduce motion vectors. However, the revisit time of SAR satellites is often too low to allow the clear recognition - even with satellites that are in a polar near orbit.

In this context, we present a multi-satellite approach to overcome this drawback. We use TerraSAR-X (TS-X) and RADARSAT-2 (RS2) acquisitions with time separation of only a few hours. The short time separation allows estimating the drift by pattern matching techniques with a high reliability.

Our test deals with images acquired during a joint campaign with the Office of Naval Research (ONR) with the research vessel RV Sikuliaq in the Beaufort Sea from October to November 2015. For estimating the sea ice drift automatically from these images, we implemented a phase correlation (PC) technique with a hierarchical motion estimation framework. The output drift field has a resolution of $1 \mathrm{~km} \times 1 \mathrm{~km}$ and reveals small variations within the sea ice motion.

The test results show that the processor is able to handle TerraSAR-X X-Band and RADARSAT-2 C-Band data and

\begin{tabular}{|c|c|c|c|c|c|c|}
\hline $\begin{array}{c}\text { Data } \\
\text { set }\end{array}$ & $\begin{array}{c}\text { Acquisition } \\
\text { date }\end{array}$ & $\begin{array}{c}\text { Acquisition } \\
\text { time }\end{array}$ & Mission & Mode & $\begin{array}{c}\text { incidence angle } \\
\text { range }\end{array}$ & Orbit \\
\hline 1 & $2015 / 10 / 23$ & $02: 11$ & TS-X & ScanSARWide & $15.3^{\circ}-40.6^{\circ}$ & Ascending \\
\hline 2 & $2015 / 10 / 23$ & $03: 56$ & RS2 & ScanSARWide A & $19.6^{\circ}-49.4^{\circ}$ & Ascending \\
\hline 3 & $2015 / 10 / 23$ & $19: 40$ & TS-X & ScanSARWide & $31.1^{\circ}-46.9^{\circ}$ & Descending \\
\hline 4 & $2015 / 10 / 24$ & $01: 53$ & TS-X & ScanSARWide & $15.3^{\circ}-40.6^{\circ}$ & Ascending \\
\hline 5 & $2015 / 10 / 24$ & $17: 23$ & RS2 & ScanSARWide A & $19.6^{\circ}-49.4^{\circ}$ & Descending \\
\hline
\end{tabular}

Table 1: Selected SAR images acquired during the Sikuliaq research cruise in fall 2015 over Beaufort Sea. All acquisitions are in HH polarization. 
therefore makes possible filling coverage gaps as well as observing fast sea ice dynamics on shorter time scales.

The implemented processor is foreseen to be integrated into the near-real time (NRT) Toolbox SAINT of the German Aerospace Center (DLR). It is intended to be part of the operational data processing chain at DLR Ground Station Network (GSN) sites.

\section{SIKULIAQ CRUISE}

The Sikuliaq research cruise took place in western Arctic Ocean in the fall of 2015. Its major goal was to observe the fall ice advance and the interactions with winds and waves. Therefore, a series of more than 400 SAR images were provided by the US National Ice Center, the German Aerospace Center (DLR), the Bedford Institute of Oceanography, the Department of Fisheries and Oceans Canada, CSTARS at the University of Miami and the Italian Consilio Nazionale per la Ricerca (CNR).

In our work, we use the SAR images for estimating the sea ice motion. Exemplarily, we show estimation results of images acquired on 23rd and 24th October 2015. Details of the images are listed in Table 1.

\section{PROCESSOR}

There is a variety of different approaches to estimate sea ice drift vectors from consecutive images, most of them categorized into (1) optical flow / gradient based motion tracking approaches or (2) pattern matching or (3) feature tracking [1][2]. While each method has benefits and drawbacks, pattern matching approaches have advantages for the analysis of images of different sources and have thus been applied in this study. The basic workflow of the pattern matching algorithm is depicted in Figure 1. It consists of the generation two co-registered image patches and the calculation of a cost function that represents the similarity of the two patches. Shifting of one of the patches alters the cost function and a particular shift minimizes the cost function, expressing maximal similarity. We apply phase correlation to determine the shift of the image patch and thus obtain a drift vector $(u, v)$. Phase correlation has been first applied in [3] and was found to be tolerant to brightness differences between the patches and comparatively insensitive to rotation. The method is thus widely used in image analysis and is particularly well-suited for the application presented in this paper.

\subsection{Resolution pyramid}

The choice of a suitable patch size is cumbersome. Large patches might cover areas with inhomogeneous drift movements and thus yield inconclusive drift estimations. Small patches might not cover large enough areas to find reliable displacements vectors. A common approach to address this problem is a nested iterative method with large
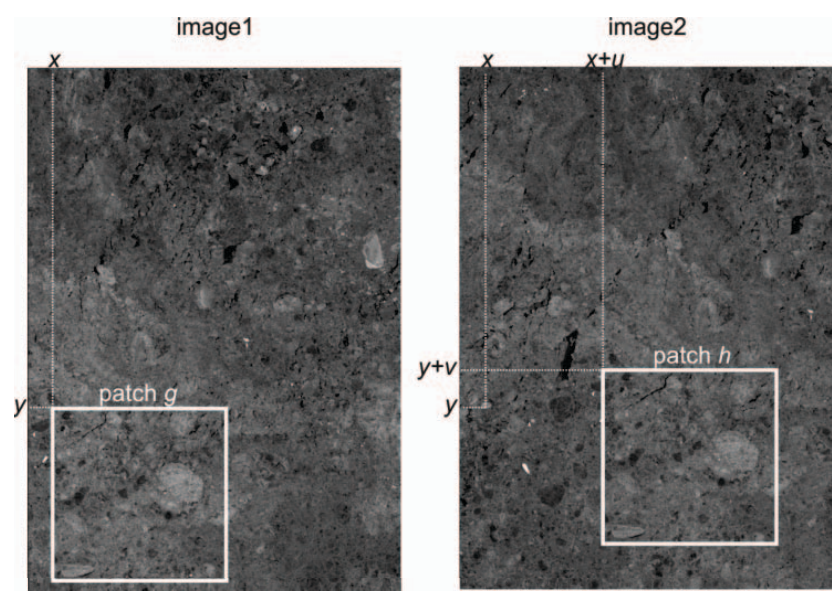

Figure 1: Principle of pattern matching recognition.

patches to find far displacement and a subsequent stepwise downsizing of the patch size in following analysis iterations. Especially for the biggest patches, a downsampled image with lower resolution is sufficient. This saves calculation time which is a benefit for the intended operational use at the DLR GSN.

In our approach, image patches yield constant dimensions of $N \times N$ pixels. We introduce the following notation:

$g\left(x_{i}, y_{i}\right)=$ image $1(x+i, y+j) \quad$ with $i=1 \ldots N, j=1 \ldots N$

$h\left(x_{i}, y_{i}\right)=$ image $2(x+i+u, y+j+v)$ with $i=1 \ldots N, j=1 \ldots N$

$(u, v)$ represents the drift vector estimated in the last iteration step. In the first iteration, there is $u=0, v=0$.

\subsection{Estimation of a drift vector}

The core of our drift vector estimation processor is a complex Fast Fourier Transform (FFT) implemented in C. After creating a pair of image patches as described in Section 3.2, we apply Von-Hann filtering [5] in order to avoid discontinuities at the patch margins. Then, we calculate the normalized image cross spectrum

$$
P C(u, v)=\mathfrak{I}^{-1}\left(\frac{\mathfrak{T}(g) \cdot \mathfrak{I}^{*}(h)}{\left|\mathfrak{I}(g) \cdot \mathfrak{\Im}^{*}(h)\right|}\right)
$$

The reverse transformation of $P C$ to the spatial domain shows a distinct peak that represents the shift of "pattern" visible in both patches.

\subsection{Update in one resolution level}

Inspired by the „consistency check" in [2] that comprises a re-estimation of the drift vectors after permuting the input images, we update the drift vector estimation (as described in Section 3.2) multiple times in each resolution level; With every iteration, patch $h$ is newly created but with updated 


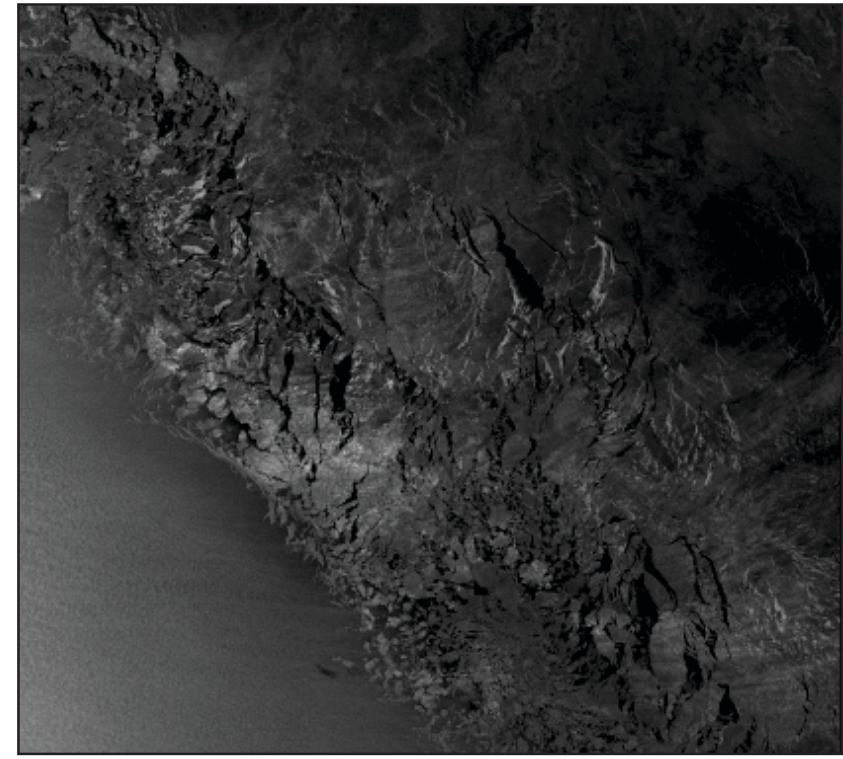

Figure 2: Section of data set 2 (RS2 acquisition over the marginal ice zone in Beaufort Sea on 2015/10/23 at 03:56 UTC). The homogeneous area in the south west (bottom left) shows open water, other areas are covered with ice.

Ship position within the footprint at N73.703 ${ }^{\circ}$, W159.047 .

vector components $u$ and $v$, transformed, and finally $P C$ is re-calculated. By doing so, the estimated drift vector becomes more accurate. This procedure is repeated as long as the updates are above displacement of one pixel.

\section{TEST RESULTS}

The processor is tested with a series of SAR images collected during the Sikuliaq research cruise. Exemplarily, Figure 4 shows the resulting drift vector field of two SAR images depicted in Figure 2 and 3 (data set 2 and 1 in Table 1).

Obviously, the sea ice drifted in the direction of the open water (i.e. east to west) with an average velocity of $1.6 \mathrm{~km} / \mathrm{h}$. At the marginal ice zone, the drift speed was generally higher than in the more compact ice in the north east. In consequence, new open leads appeared and existing open leads grew. These areas become visible in Figure 4 (a) by jumps in the color coding from dark blue to cyan (approx. at $\mathrm{N} 73.6^{\circ}, \mathrm{W} 160^{\circ}$ ). This coincides well with the observed ice situation.

In some areas, automatic sea ice drift estimation is not possible. In particular, in case of strong changes within the sea ice, but also within homogeneous areas with few pronounced surface features, pattern recognition fails. However, the correlation coefficient quantifies the quality of the estimation. In the present case, areas of low correlation values (correlation values below a threshold of 0.025 ) are mapped in white color and excluded for drift calculation. They mainly occur in the open water area.

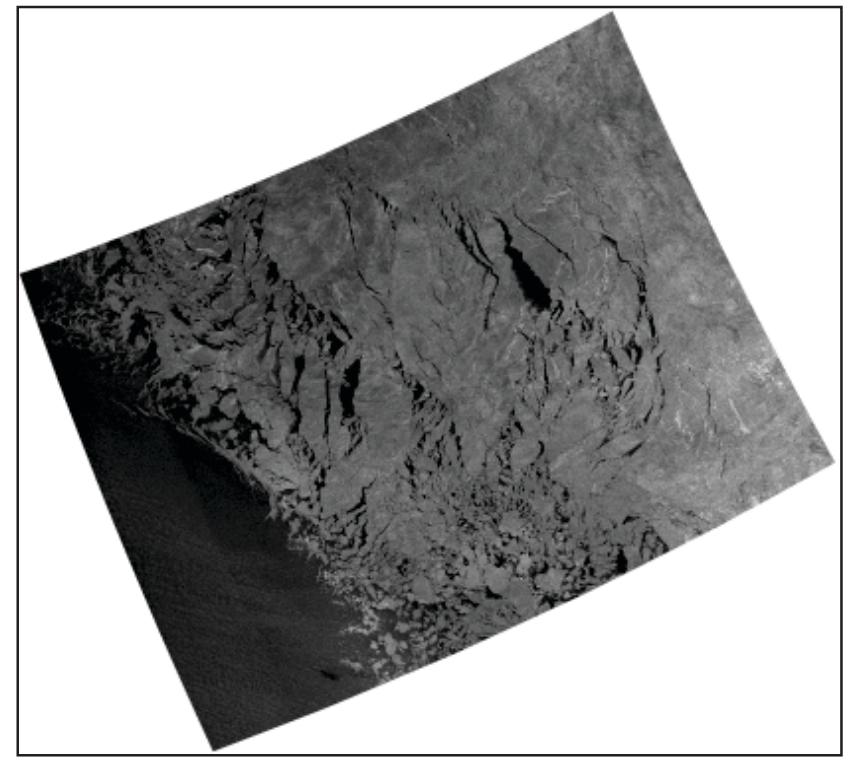

Figure 3: Data set 1 (TS-X acquisition over Beaufort Sea on 2015/10/23 at 02:11 UTC), co-registrated to data set 2. Ship position within the footprint at N73.871 ${ }^{\circ}$, W159.073 .

Correspondingly, Figure 4 (b) shows the resulting drift vector field derived from data sets 2 and 3 . Due to the location of data set 3 further north, it captures motion that occurred further into the ice zone. Here, the average sea ice velocity is reduced to $0.5 \mathrm{~km} / \mathrm{h}$. The average drift direction changed from east to south east.

In the follow up data sets 4 and 5 that are both collocated to data set 1, advancing sea ice deformation makes an observation of the drift impossible, for both, the processor and manual inspection of the SAR images. The drift vectors throughout yield a correlation value below the given threshold.

\section{CONCLUSIONS}

In this paper, we present first test results of a sea ice drift estimation processor which works on pairs of consecutive SAR images from different sensors and operating in different bands and resolutions. The combination of different SAR sensors improves significantly the observation frequency of ice infested waters. This is crucial for consistent drift estimation, as already within hours sea ice can change significantly and make pattern recognition impossible.

Our tests show that in case of strong changes within the ice, the proposed processor outputs low correlation values. For moderate changes, a high correlation is achieved. The derived sea ice drift fields are homogeneous and coincidence well with the observed ice situation. 

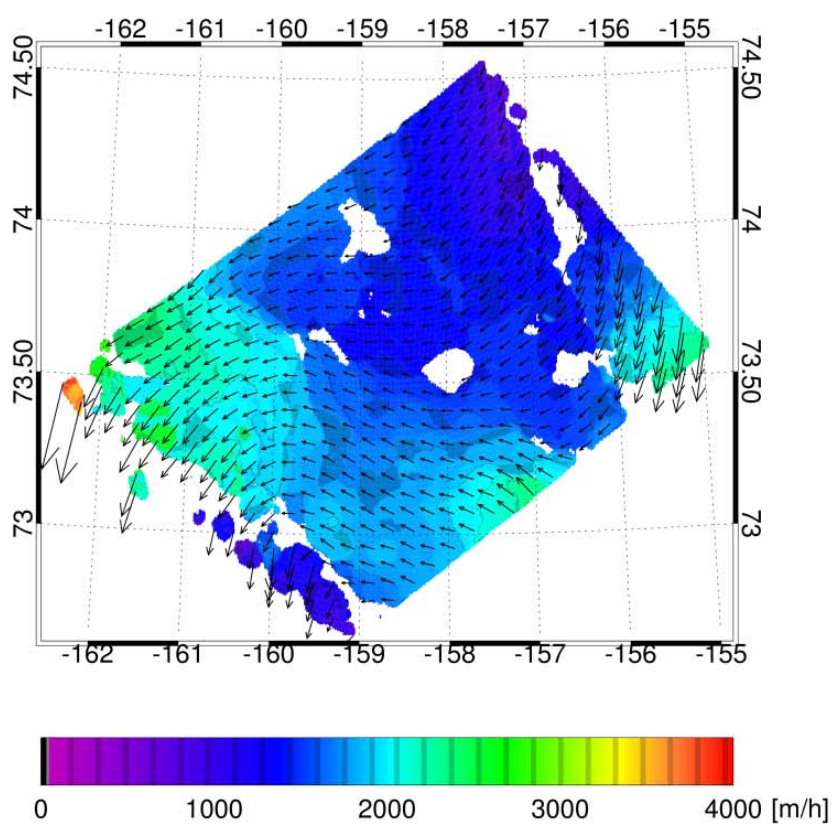

(a)

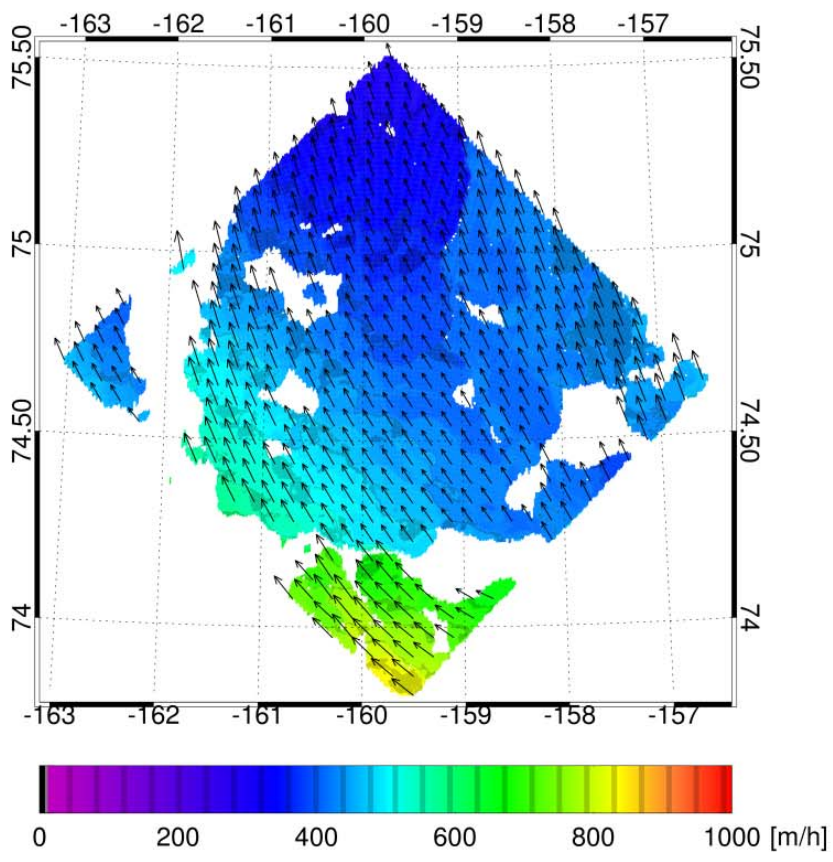

(b)

Figure 4: Output of the sea ice drift estimation processor. The estimation is based on pairs of SAR images. (a) Result after processing data set 1 and 2. (b) Result after processing data set 2 and 3. The estimated drift fields have a resolution of $1 \mathrm{~km} \times 1 \mathrm{~km}$, and reveal small changes within the ice motion e.g. the occurrence of new open leads. Areas of low correlation between the two SAR images are indicated in white color.

The results can be assimilated into drift models in order to improve the forecast accuracy. Another area of application is the integration into sea ice classification [6]. The sea ice drift fields reveal small-scale spatial changes in ice velocity and direction and thus can be used to identify compressed ice, sea ice ridges and to border ice sheets that show individual motion. In future works, we want to investigate the benefit of sea ice drift fields when integrated into sea ice classification for improved characterizations.

\section{ACKNOWLEGEMENTS}

TS-X and RS2 dataset was acquired in context of ONRProject. RS2-products (C) MDA.

\section{REFERENCES}

[1] J. Karvonen. Operational SAR-based sea ice drift monitoring over the Baltic Sea. In: Ocean Science 8.4: 473. 2012.

[2] T. Hollands. Motion tracking of sea ice with SAR satellite data. Diss. Universität Bremen, 2012.

[3] E. De Castro, C. Morandi. "Registration of translated and rotated images using finite Fourier transforms." IEEE Transactions on Pattern Analysis \& Machine Intelligence 5 (1987): 700-703.
[4] D. Hamidi, S. Lehner, T. König, A. Pleskachevsky. On the sea ice motion estimation with synthetic aperture radar. In: Proc. 4th TerraSAR-X Meeting (pp. 1-10). 2011.

[5] R. B. Blackman, J. W. Tukey. Particular pairs of windows. In: The measurement of power spectra, from the point of view of communications engineering. Dover, New York 1959, S. 95-101. 1959.

[6] R. Ressel, A. Frost, S. Lehner. „A neural network-based classification for sea ice types on X-band SAR images.” IEEE Journal of Selected Topics in Applied Earth Observations and Remote Sensing 8.7 (2015): 3672-3680. 